\title{
Photographic positions for the first eight satellites of Saturn ${ }^{\star, \star \star}$
}

\author{
C.H. Veiga and R. Vieira Martins \\ Observatório Nacional, Rua Gal. José Cristino 77, 20921-400 Rio de Janeiro, Brazil \\ e-mail: cave@on.br or rvm@on.br
}

Received January 11; accepted July 23, 1999

\begin{abstract}
Astrometric positions of the first eight Saturnian satellites obtained from 138 photographic plates taken in 30 nights in the years 1982 to 1988 are presented. All positions are compared with those calculated by the theory TASS1.7 (Vienne \& Duriez 1998). The observed minus calculated residuals give rise to standard deviations smaller than 0.3 .
\end{abstract}

Key words: astrometry — planets and satellites — Saturn

\section{Introduction}

There are many thousands of astrometric positions of the first eight Saturn satellites. Stugnell \& Taylor (1990) present a catalogue of ground-based observations taken between 1874 and 1989, with more than 51000 observations. Later, Harper \& Taylor (1994) analyze an extensive catalog of these positions, Hyperion excluded. The theoretical positions were computed using the theory of the motion of the Saturn satellites (Harper \& Taylor 1993). From the residuals of more than 9000 photographic intersatellite positions made by 46 different authors, it is observed that about $20 \%$ are larger than $00^{\prime \prime} 3$, about $30 \%$ lay between 0.2 and 0.3 , and the remaining $50 \%$ are smaller than 0.2 . As remarked by the authors, the data sets for Mimas and Hyperion are particularly sparse.

In 1982 we started a systematic program of astrometric observations of satellites of the Jovian planets. Our results about the Uranus and Neptune systems were published in previous papers (see for example Veiga \& Vieira Martins $1995,1998)$. In this, paper we report the results from 138 photographic plates of the Saturnian system carried

\footnotetext{
* Based on observations made at Laboratório Nacional de Astrofísica/CNPq/MCT-Itajubá-Brazil. Please send offprint requests to C.H. Veiga.

** Table 2 is only available at the CDS via anonymous ftp to (130.79.128.5) cdsarc.u-strasbg.fr or via http://cdsweb.ustrasbg.fr/Abstract.html
}

out during 30 nights distributed in 12 observational missions, in the period from 1982 to 1988 . The positions for the first eight satellites are given. This set constitutes the totality of our Saturnian photographic observations. In 1989 we swept to a CCD detector and the results from this observations will be published in a forthcoming paper.

The present paper is organized as follows. In Sect. 2 the observations and reduction procedures are described and the differential coordinates of seven Saturn satellites referred to Titan are presented. In Sect. 3 our results are compared with theoretical calculated positions, as given by the theory TASS1.7 (Vienne \& Duriez 1995; Vienne \& Duriez 1998) for the eight major satellites.

\section{The observations, measurements and reductions}

All observations were made at the Cassegrain-focus of the $1.6 \mathrm{~m}$ Ritchey-Chretien reflector of the Laboratório Nacional de Astrofísica at Brazil (geographical longitude: $-3^{\mathrm{h}} 02^{\mathrm{m}} 19^{\mathrm{s}}$, latitude: $-22^{\circ} 32^{\prime} 04^{\prime \prime}$ and altitude: $1872 \mathrm{~m}$ ). The focal length of the Cassegrain combination is $15.8 \mathrm{~m}$, which results in a plate scale of $13^{\prime \prime} / \mathrm{mm}$ at the focal plane.

Photographic plates with Kodak emulsions $\mathrm{IIaO}, 103 \mathrm{aO}$, IIaF and IIIaJ and with dimensions $26 \times 21 \operatorname{arcmin}^{2}$ were used. All plates were hypersensitized and the exposure times have varied from 3 to 15 minutes. The long exposure times for many plates ( $57 \%$ have 10 or 12 minutes of exposure time) were used since the initial goal was to observe the faint satellites in the Lagrangian points of Thetys and Dione. The positions for these satellites will be published in a forthcoming paper. No filter was used. Figure 1 presents one of our images of the Saturn's system. In the figure in order to bring up the images of the faint satellites near Saturn, a gradient filter was used.

Our images of Saturnian system present some peculiar features. All Saturn images are saturated and not measurable. The images of Titan and Rhea are also saturated on the long exposure time plates. To avoid the diffraction 


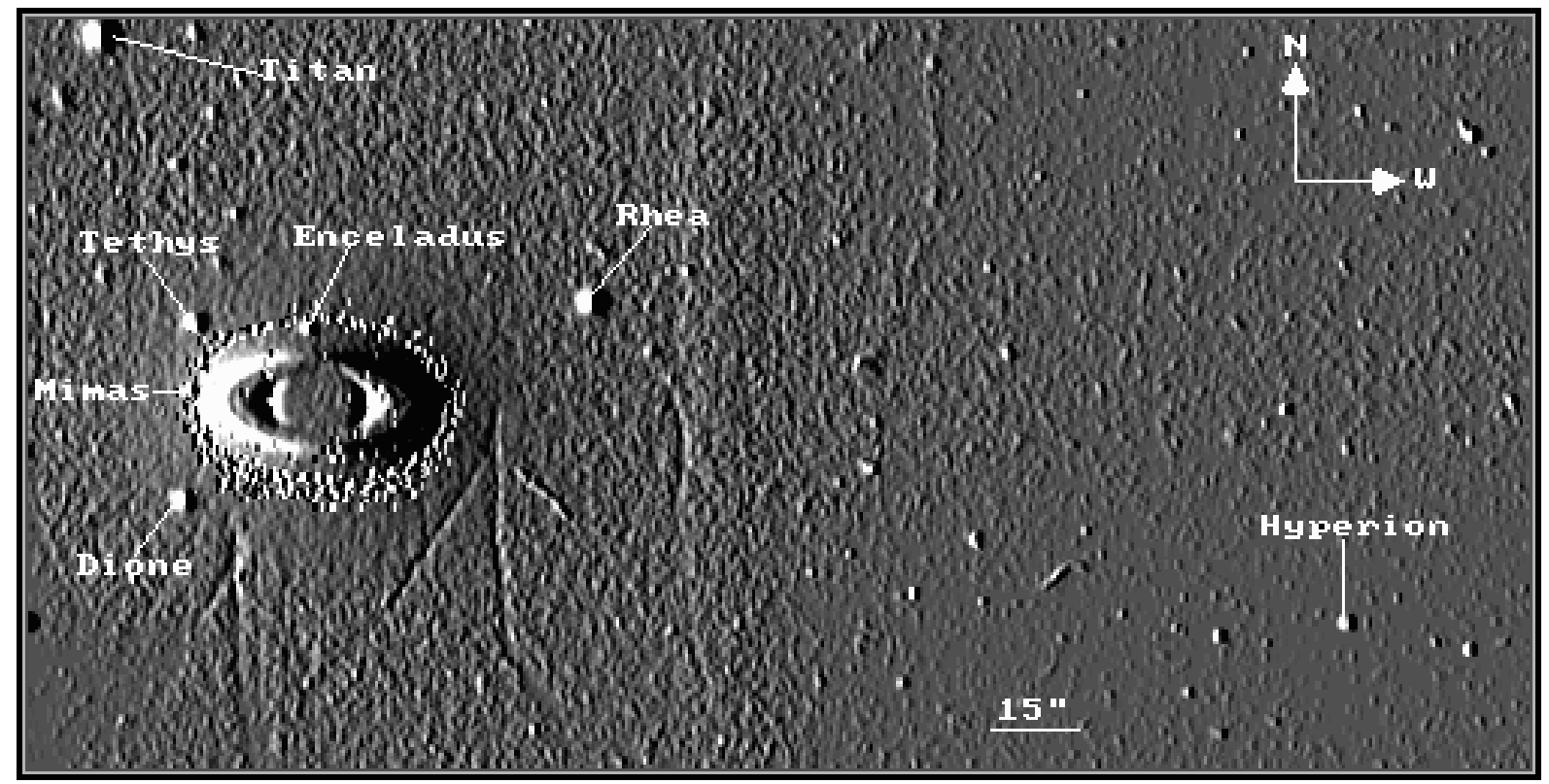

Fig. 1. Region of a photographic plate taken at $07 / 30 / 1988,1^{\mathrm{h}} 19^{\mathrm{m}} 0^{\mathrm{s}}$ UT. The emulsion is IIao and the exposure time was $3^{\mathrm{m}}$. The distance of Iapetus from Saturn is $8^{\prime} 35^{\prime \prime}$ in the East direction. Therefore, it is not in this region of the plate. For the same reason, the GSC stars cannot be seen

Table 1. Number of positions referred to Titan and of observed nights for Saturn satellites. The total number of observed plates and nights are 138 and 30 , respectively

\begin{tabular}{lcc}
\hline \multirow{2}{*}{ Satellite } & \multicolumn{2}{c}{ Number of } \\
Positions & Nights \\
\hline Mimas & 45 & 16 \\
Enceladus & 104 & 26 \\
Tethys & 102 & 28 \\
Dione & 134 & 29 \\
Rhea & 134 & 30 \\
Hyperion & 132 & 30 \\
Iapetus & 119 & 27 \\
\hline
\end{tabular}

cross in these saturated images, an eight round apertures mask was placed between the secondary mirror supporting vanes. For the plates taken with long exposure time, the images of Mimas and Enceladus are immersed in the light scattered by the planet and its bright rings. For many plates, the images are elongated probably due to guiding problems.

The number of positions of Mimas is smaller than those for the other satellites. This satellite is very difficult to observe due to its small distance from the planet and the bright rings. Table 1 presents the number of Titan's referred positions and the number of observed nights are presented for each satellite. Figure 2 shows the histogram of the number of plates with respect to the epoch of the observations. Each bar corresponds to one of the 12 observational missions.

The digitized images of the satellites and stars were obtained with the PDS 1010A of the Observatório

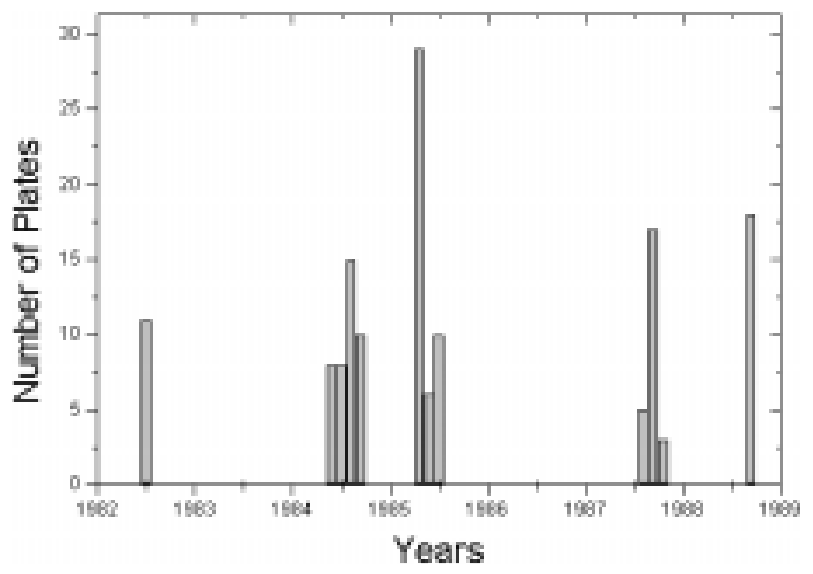

Fig. 2. Histogram of the photographic plates of Saturn with respect to time. Each bar corresponds to an observational mission

Nacional-Brazil. For the image scans a square slit which side had $20 \mu$ was used. To find the center of these digitized images, the ASTROL routines package (Colas \& Serrau 1993) was employed. A two-dimensional Gaussian fitting on a small circular area around the image, in which the background was removed by a second-degree polynomial, was used to determine each center. The errors upon the centering procedure where at $00^{\prime \prime} 01$ for the satellites and field stars, while at $0{ }^{\prime \prime} 02$ for Mimas. To ensure a robust centering for Mimas images, the image position has been computed for 10 different centers of circular area around the satellite. The differences between the computed positions were smaller than 0.01 . 

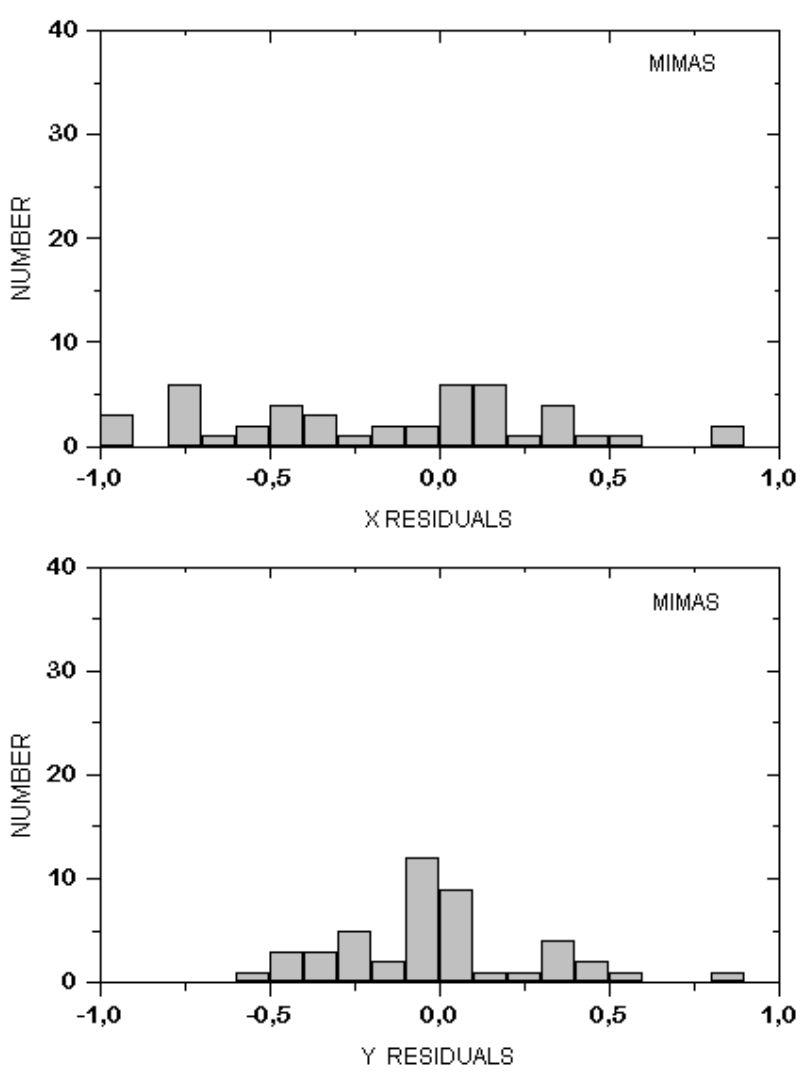

Fig. 3. Histogram for the right ascension $(X)$ and declinations $(Y)$ residuals of the Saturnian satellites referred to Titan

The astrometric calibration was performed using the method presented in Vieira Martins et al. (1996) (see also Assafin et al. 1997). It consists on the setting of an astrometric catalogue for the stars on the plate, using the Guide Star Catalogue (GSC) corrected by the PPM Catalog. It can be remarked that, by they relative nature, the present astrometric determinations would not be improved through a Hipparcos tied GSC correction. The mean number of GSC measurable stars around Saturn was 24 per plate. However, for some plates we have few GSC measurable stars or they were not well distributed around Saturn. For every plate, the RMS of the residuals for the plate stars is about 0 .' 16 in the right ascension and declination directions. In order to evaluate the accuracy of the astrometric calibration, we compared the equatorial coordinates of GSC stars, plate by plate, in a same mission. The RMS of the differences is around $00^{\prime \prime} 07$. The difference between these errors indicates that an important source of errors is just the bad theoretical positions of the GSC stars positions.

The theoretical positions of Saturn were calculated using DE403 (Standish et al. 1995). To compute the light travel time the topocentric distance from the center of Saturn was adopted, since the error on the satellite position due to this approximation is smaller than 0'006 (Harper \& Taylor 1994). However, for Iapetus the topocentric distance of the satellite was used.
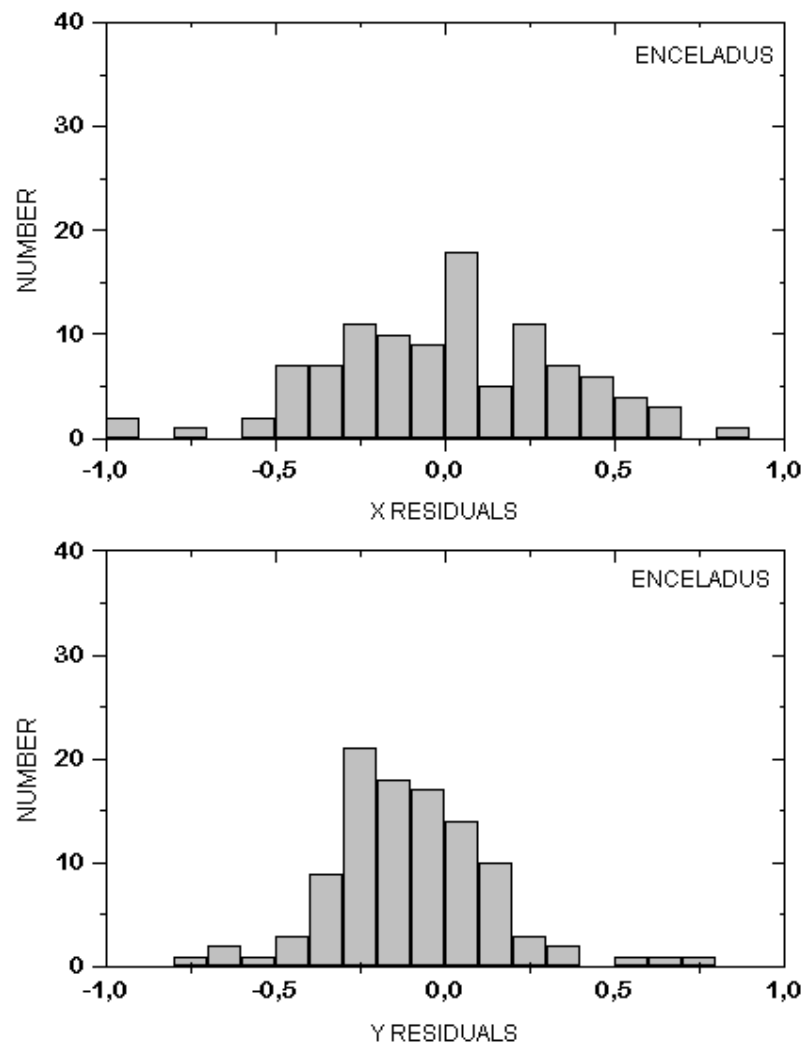

Fig. 3. continued
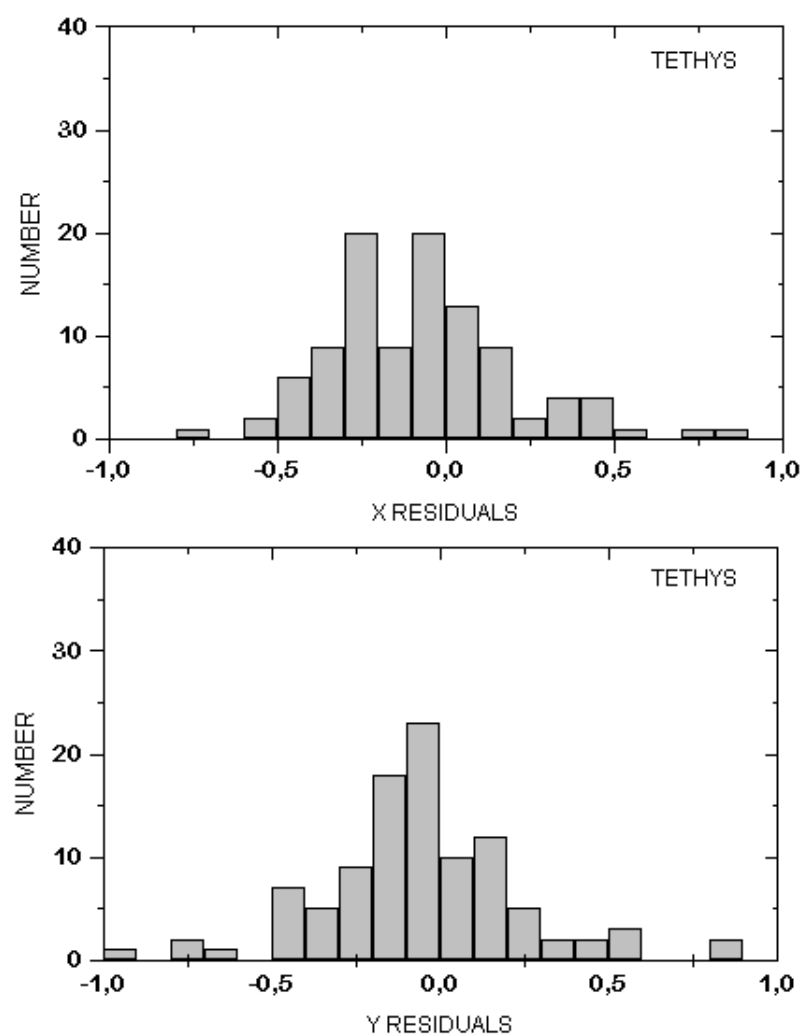

Fig. 3. continued 

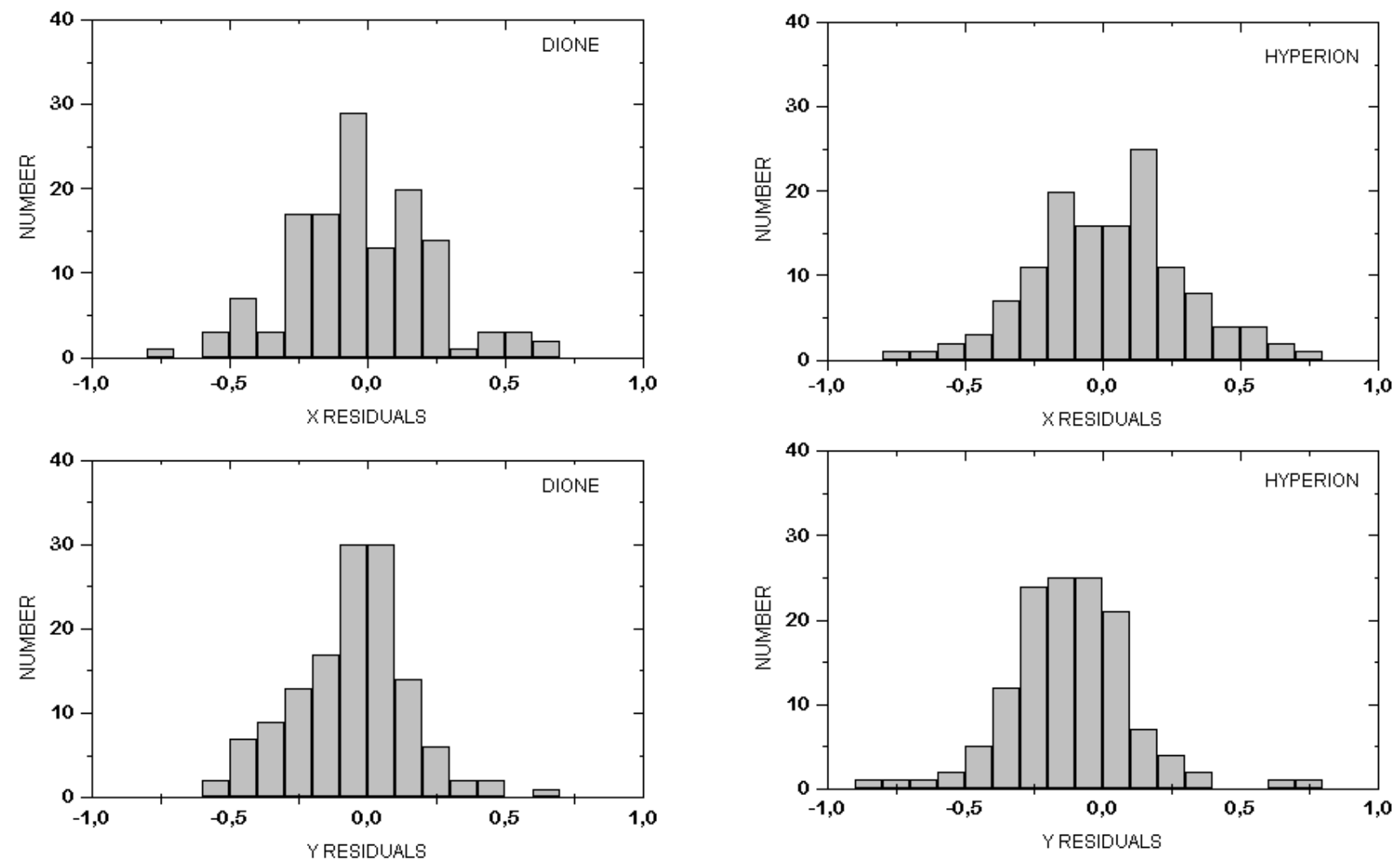

Fig. 3. continued

Fig. 3. continued
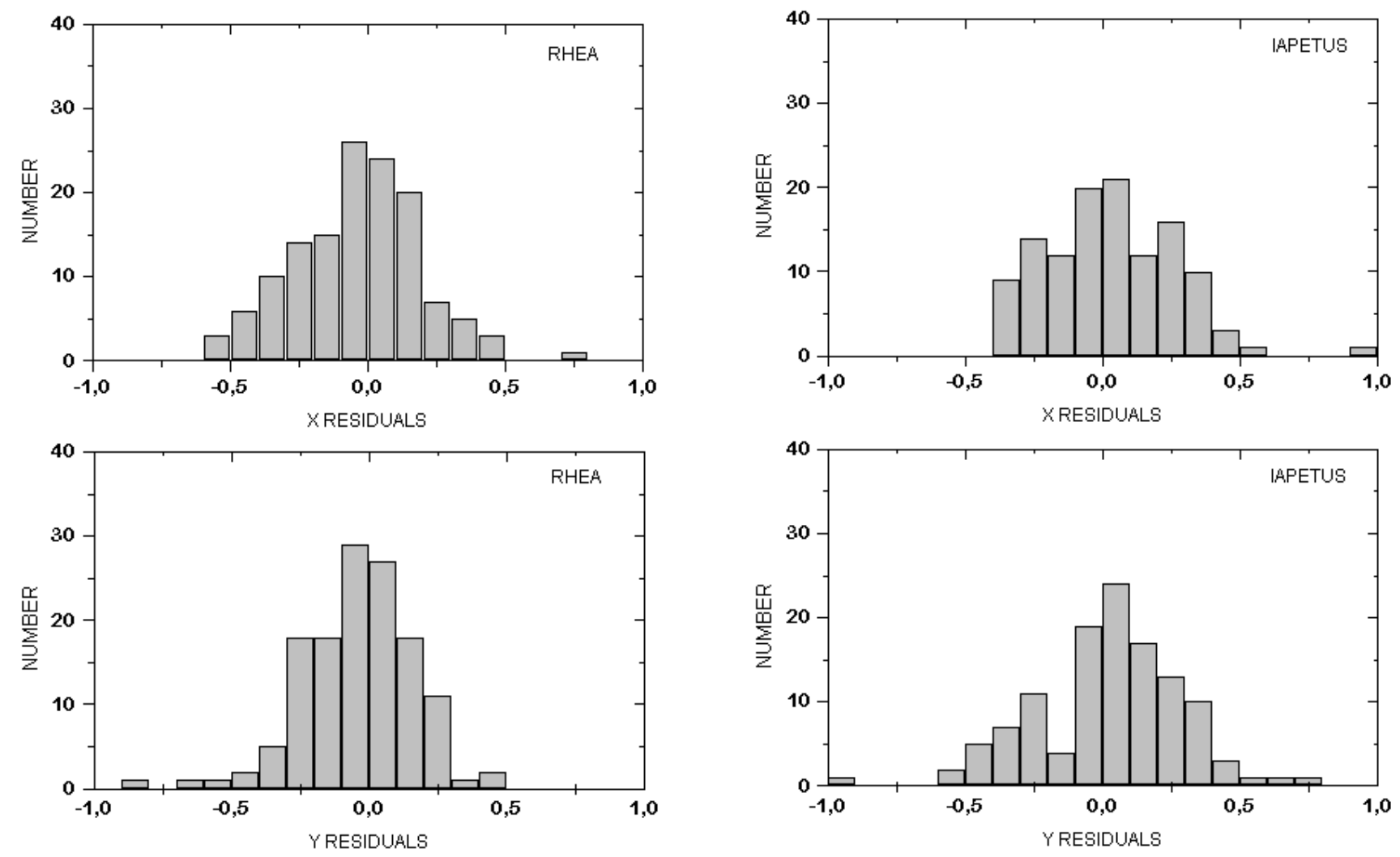

Fig. 3. continued

Fig. 3. continued 
Table 3. The observed minus calculated means and standard deviations, in arcseconds, referred to Titan. $N$ is the number of positions referred to Titan

\begin{tabular}{llrl}
\hline Satellite & $N$ & \multicolumn{2}{c}{ Means and Standard deviations } \\
& & \multicolumn{1}{c}{$(\mathrm{O}-\mathrm{C})_{x}(\sigma)$} & $\overline{(\mathrm{O}-\mathrm{C})_{y}}(\sigma)$ \\
\hline Mimas & $(51)$ & $-0.11(0.39)$ & $-0.07(0.28)$ \\
Enceladus & $(105)$ & $0.00(0.33)$ & $-0.09(0.24)$ \\
Tethys & $(102)$ & $-0.08(0.27)$ & $-0.05(0.30)$ \\
Dione & $(134)$ & $-0.02(0.24)$ & $-0.06(0.21)$ \\
Rhea & $(134)$ & $-0.04(0.21)$ & $-0.05(0.20)$ \\
Hyperion & $(134)$ & $0.02(0.27)$ & $-0.10(0.23)$ \\
Iapetus & $(118)$ & $0.02(0.22)$ & $-0.02(0.18)$ \\
\hline
\end{tabular}

In Table 2 (accessible in electronic form) are listed our positions of the Saturn satellites related to Titan. The data are presented in the same format used in the catalogue of Strugnell \& Taylor (1990). The reference system is defined by the mean equator and equinox J2000.

\section{Comparison between the observed and the theoretical positions}

We compared the measured and theoretical positions of the Saturnian satellites. The theoretical positions for Mimas, Enceladus, Tethys, Dione, Rhea, Titan, Hyperion and Iapetus were calculated using TASS1.7 (Vienne \& Duriez 1992, 1998; Duriez \& Vienne 1997a, 1997b). For the residuals $(\mathrm{O}-\mathrm{C})$, the number of positions, the means and the standard deviations for each satellite referred to Titan are presented in Table 3. Only the residuals smaller than $1^{\prime \prime}$ were considered. The residuals referred to Saturn were not computed because the center of the planet could not be determined from its saturated image.

It can be noticed that, for Mimas, there are few positions of the satellite and the mean and the standard deviation in the right ascension direction are comparatively large. As mentioned above, this follows from difficulty to observe this satellite due to its small distance from the planet bright rings. The right ascension standard deviations for Enceladus share nearly the same problem but in general its elongation is larger and its right ascension residuals are smaller than Mimas' are.

The residuals for the other satellites have all close values. For Hyperion the residuals in right ascension are larger than the residuals for the others external satellites. This value agrees with the results in Table 9 in Duriez \& Vienne (1997). For almost all satellites, one can observe that, in general, the residuals in declination are smaller than in right ascension. This happens also for the photographic positions of Saturn satellites in Table 2 in Harper \& Taylor (1994). Probably, this is due to errors in the guiding procedure during the observations.

The positions of the satellites were taken in respect to Titan and this choice might have introduced some
Table 4. The observed minus calculated means and standard deviations, in arcseconds, for intersatellites positions. $N$ is the number of intersatellite calculated positions

\begin{tabular}{llrr}
\hline Satellite & $N$ & \multicolumn{2}{c}{ Means and Standard deviations } \\
& & \multicolumn{1}{c}{$\overline{(\mathrm{O}-\mathrm{C})_{x}}(\sigma)$} & \multicolumn{1}{c}{$\overline{(\mathrm{O}-\mathrm{C})_{y}}(\sigma)$} \\
\hline Mimas & $(297)$ & $-0.12(0.36)$ & $-0.03(0.28)$ \\
Enceladus & $(509)$ & $0.02(0.32)$ & $-0.04(0.25)$ \\
Tethys & $(509)$ & $-0.05(0.30)$ & $-0.03(0.30)$ \\
Dione & $(597)$ & $0.00(0.28)$ & $0.00(0.24)$ \\
Rhea & $(605)$ & $-0.03(0.25)$ & $0.03(0.24)$ \\
Titan & $(607)$ & $0.02(0.27)$ & $0.08(0.23)$ \\
Hyperion & $(599)$ & $0.04(0.29)$ & $-0.06(0.24)$ \\
Iapetus & $(653)$ & $0.02(0.23)$ & $0.03(0.20)$ \\
\hline
\end{tabular}

bias on our results. To avoid this problem we computed the intersatellite positions too that is, the relative positions between each satellite against Enceladus, Tethys, Dione, Rhea, Titan, Hyperion and Iapetus. This procedure was not applied in reference to Mimas since this satellite doesn't present good positions as seen in Table 3. These results are presented in Table 4 . The comparison between Table 4 and Table 3 shows them not be essentially different. Finally, we computed also the intersatellite positions, taking only Tethys, Dione, Rhea and Titan. The residuals are almost the same. Therefore, it is shown that the choice of Titan as reference satellite doesn't introduce any bias on our results.

Figure 3 presents the histogram of the residuals for our positions referred to Titan. It can be observed that, but for the Mimas right ascension, the residuals for the satellites have distributions similar to a normal distribution.

\section{Conclusion}

We presented the results of our photographic observations taken with a 1.6 m reflector, between 1982 and 1988 . They correspond to 138 plates obtained during 30 nights and distributed in 12 observational missions. There are 770 new positions of the major satellites of Saturn, which corresponds to 2176 intersatellite positions. These observations partially supply the absence of published positions of the Saturnian satellites after 1984. As Duriez and Vienne (1997b) pointed out, it is important not to have a gap in the temporal distribution of the observations, if we want to construct good ephemeris.

The observed minus calculated residuals in right ascension and declination are about $0 . \prime 3$, except for Mimas. The large values for the right ascension residuals of Mimas are due to the long exposure time for many of our plates. Nevertheless, such long exposure time is responsible for the good positions of Hyperion, which is the faintest of the first eight satellites of Saturn.

In 1989 we swept to a CCD detector. The results of these observations will be published in a forthcoming paper. 
Acknowledgements. We want to thank M.E. Velasco Kopp and A.H. Andrei, for their help in this work and the staff of Laboratório Nacional de Astrofísica for the assistance during our observations. We thanks also A. Vienne and M. Rapoport for the suggestions in the final version of this paper. Finally, C.H. Veiga and R. Vieira Martins thanks the CNPq-Brazil for partial support of this work.

\section{References}

Assafin M., Vieira Martins R., Andrei A.H., 1997, AJ 113, 1451

Colas F., Serrau M., 1993, Astrol and Interpol version 3.10. Édition du Bureau des Longitudes

Duriez L., Vienne A., 1997a, A\&A 324, 366-380

Duriez L., Vienne A., 1997b, in Dynamics and Astrometry of Natural and Artificial Celestial Bodys, Wytrzyszczak
I.M., Lieske J.H., Feldman R.A. (eds.). Kluwer, Dordrecht, pp. $177-182$

Harper D., Taylor D.B., 1993, A\&A 268, 326-349

Harper D., Taylor D.B., 1994, A\&A 284, 619-628

Standish E.M., Newhall X.X., Williams J.G., Folkener W.P., 1995, "JPL Planetary and Lunar Ephemeris DE403/LE403", JPL IOM 314.10-127

Strugnell P.R., Taylor D.B., 1990, A\&AS 83, 289-300

Veiga C.H., Vieira Martins R., 1995, A\&AS 113, 557-560

Veiga C.H., Vieira Martins R., 1998, A\&AS 131, 291-293

Vieira Martins R., Veiga C.H., Assafin M., 1996, in Dynamics Ephemerides and Astrometry of the Solar system, Ferraz Mello S., Morando B. and Arlot J.E. (eds.). Kluwer, Dordrecht, pp. 419-422

Vienne A., Duriez L., 1998 (personal communication)

Vienne A., Duriez L., 1995, A\&A 297, 588-605

Vienne A., 1999 (personal communication) 